\title{
Allelic variation and prevalence of serum opacity factor among the Streptococcus suis population
}

Correspondence
Tsutomu Sekizaki
sekizaki@affrc.go.jp
Received 8 November 2007 Accepted 5 December 2007

\author{
Daisuke Takamatsu, ${ }^{1}$ Makoto Osaki, ${ }^{1}$ Prasit Tharavichitkul, ${ }^{2}$ Shinji Takai ${ }^{3}$ \\ and Tsutomu Sekizaki ${ }^{1,4}$
}

\author{
${ }^{1}$ National Institute of Animal Health, Tsukuba, Ibaraki 305-0856, Japan \\ ${ }^{2}$ Faculty of Medicine, Chiang Mai University, Chiang Mai 50200, Thailand \\ ${ }^{3}$ School of Veterinary Medicine and Animal Sciences, Kitasato University, Towada, Aomori \\ 034-8628, Japan \\ ${ }^{4}$ United Graduate School of Veterinary Sciences, Gifu University, Gifu 501-1193, Japan
}

\begin{abstract}
Serum opacity factor of Streptococcus suis (OFS) has recently been identified as a virulence determinant of an S. suis strain. In this study, we investigated the prevalence and variations of the ofs gene among 108 S. suis isolates from diseased and healthy pigs, and human patients. PCR screening and sequencing analysis showed that besides the ofs gene reported already (designated type 1), there were three allelic variants of ofs (designated types 2 to 4 ). Type- 1 and type- 2 ofs genes were expected to encode functional OFS, and SDS extracts of the isolates with type-1 ofs and type-2 ofs opacified horse serum. Culture supernatants of the isolates with type-2 ofs also showed strong serum opacification activity. In contrast, type-3 ofs was interrupted by a point mutation and type-4 ofs was disrupted by either insertion of an IS element or genetic rearrangement, and therefore the SDS extracts and culture supernatants of the isolates with type- 3 ofs and type- 4 ofs did not show serum opacification activity. Regardless of their origins, approximately $30 \%$ of the isolates possessed functional OFSs, although type-2 ofs was found only in three isolates from healthy pigs. Multilocus sequence typing analysis showed that most of the isolates with type- 1 ofs belonged to the sequence type (ST)1 complex, and most of the isolates with type-3 ofs and type-4 ofs belonged to the ST27 complex. The isolates with type-2 ofs were not assigned to a major ST complex. These results suggest that type-1 OFS contributes to the virulence of a limited number of $S$. suis isolates, i.e. those of the ST1 complex type, whereas other S. suis may not possess this category of virulence factor; the importance of type-2 OFS is obscure.
\end{abstract}

\section{INTRODUCTION}

Streptococcus suis is an important pathogen associated with a wide range of diseases in pigs, including meningitis, septicaemia, arthritis, pneumonia, endocarditis and sudden

Abbreviations: EF, extracellular factor; MLST, multilocus sequence typing; MRP, muramidase-released protein; OFS, opacity factor of Streptococcus suis; SOF, serum opacity factor of Streptococcus pyogenes; ST, sequence type.

The GenBank/EMBL/DDBJ accession numbers for the sequences of the ofs loci of S. suis NIAH 11433, DAT299, DAT246, DAT252 and MNCM51 are AB325706, AB325707, AB325708, AB325709 and AB325710, respectively, and for the new allele sequences of aro $A$, cpn60, dpr, gki, mutS, recA and thrA are AB330897-AB330899, AB330900-AB330907, AB330908-AB330909, AB330910AB330915, AB330916-AB330922, AB330923-AB330927 and AB330928-AB330932, respectively.

A table of isolates and a figure showing Southern blot hybridization analysis are available as supplementary material with the online version of this paper. death (Staats et al., 1997). S. suis is also known as a zoonotic agent affecting people in close contact with diseased pigs or their products (Arends \& Zanen, 1988; Chang et al., 2006; Ye et al., 2006; Takamatsu et al., 2008).

Despite increasing research in recent years, knowledge of the virulence determinants of $S$. suis remains limited. The polysaccharide capsule of $S$. suis serotype 2 is proven to be one of the major virulence determinants (Smith et al., 1999). However, not all encapsulated serotype 2 strains are virulent and there is variation in the degrees of virulence among the strains (Vecht et al., 1989, 1992). Published studies have also reported several putative virulence factors, including the muramidase-released protein (MRP), the extracellular factor (EF) and suilysin (Jacobs et al., 1994; Vecht et al., 1991). In contrast to $\mathrm{MRP}^{-} \mathrm{EF}^{-}$serotype 2 strains from Europe, serotype 2 strains expressing the $136 \mathrm{kDa} \mathrm{MRP}$ and the $110 \mathrm{kDa}$ EF were highly virulent (Vecht et al., 1991, 1992). Therefore, these factors were thought to contribute to the pathogenesis of S. suis. However, because many virulent 
isolates lacking these factors have also been isolated from clinical cases in North America (Gottschalk et al., 1998), the significance of these factors in the pathogenesis of S. suis is still unclear.

Recently, a serum opacity factor of $S$. suis (OFS) has been identified as a novel virulence determinant of $S$. suis serotype 2 (Baums et al., 2006). This protein has the typical structural features of Gram-positive surface proteins, which consist of a putative $\mathrm{N}$-terminal signal sequence, a large $\mathrm{N}$-terminal domain, repetitive sequence elements and a C-terminal LPXTG anchor motif (Baums et al., 2006). Particularly, the $\mathrm{N}$-terminal domain is homologous to the corresponding region of the serum opacity factor of Streptococcus pyogenes (SOF), and the domain has been shown to have serum opacification activity (Baums et al., 2006). Disruption of ofs resulted in a severe attenuation of virulence when investigated in porcine experimental models, suggesting the significance of OFS in the pathogenesis of S. suis (Baums et al., 2006). However, it is unknown whether this factor is widely present among $S$. suis strains, as only one strain was used for the reported study.

In this study, we investigated the prevalence of the ofs gene using 108 S. suis isolates from diseased pigs, healthy pigs and human patients. Our results showed that there were at least four distinct ofs genotypes in S. suis, and one of the genotypes was strongly associated with isolates of a genetically clonal group that is frequently isolated from pigs and humans with invasive disease throughout the world.

\section{METHODS}

Bacterial isolates and culture conditions. A total of 108 S. suis isolates were used in this study (see Supplementary Table S1 available with the online journal). Among them, 90 Asian isolates (51 from diseased pigs, 18 from healthy pigs and 21 from human patients) were from our laboratories' collection. Seventeen well-characterized North American and European strains (eight from diseased pigs, three from healthy pigs and six from human patients) were supplied by $\mathrm{M}$. Gottschalk (Faculté de Médicine Vétérinaire, Université de Montréal, Montreal, Canada). The reference strain of serotype 2 was purchased from the National Collection of Type Cultures, London, England. Bacteria were cultured in Todd-Hewitt broth or agar supplemented with $2 \%$ yeast extract (THY) (Difco Laboratories, Becton Dickinson), or Columbia agar (Difco Laboratories) with $5 \%$ horse blood in air plus $5 \% \mathrm{CO}_{2}$.
DNA methods. S. suis chromosomal DNA was extracted as described previously (Osaki et al., 2000). Ex Taq polymerase (Takara Bio) was used for PCR amplifications. For PCR-based screening of the ofs gene, primers Ofs-F (5'-GATGTGACTGTCCGCAGAGC- $3^{\prime}$ ) and Ofs-R2 (5'-AAAGTACCTGAGCTCCTACA- $3^{\prime}$ ) were used to amplify the internal segment of ofs (Fig. 1a), and reaction mixtures were prepared according to the manufacturer's instructions, except that the concentration of $\mathrm{MgCl}_{2}$ used was $2.5 \mathrm{mM}$. The program for the amplification consisted of an initial denaturation of 2 min at $96{ }^{\circ} \mathrm{C}$, 30 cycles of $20 \mathrm{~s}$ at $96{ }^{\circ} \mathrm{C}, 10 \mathrm{~s}$ at $55^{\circ} \mathrm{C}$ and $1 \mathrm{~min}$ at $72{ }^{\circ} \mathrm{C}$, and a final extension of $1 \mathrm{~min}$ at $72{ }^{\circ} \mathrm{C}$. Southern blot hybridization was performed as described previously (Sekizaki et al., 2001) with a DIG (digoxigenin) DNA labelling and detection kit (Roche). Prehybridization and hybridization were carried out at $65{ }^{\circ} \mathrm{C}$ for 2 and $16 \mathrm{~h}$, respectively. For preparation of the ofs probe, an internal fragment of ofs was amplified from the genomic DNA of NIAH 11433 using primers Ofs-pr1 (5'-AAATAAGGAAGGAGAGGTGG-3') and Ofs-pr2 (5'-ACAAGAAGAGAGAGGGCAGT- $\left.3^{\prime}\right)$, and labelled with DIG by using the DIG DNA labelling and detection kit. Sequencing of various PCR products was carried out with a BigDye terminator v3.1 cycle sequencing kit using a 3100 Genetic Analyzer (Applied Biosystems).

Sequence determination of ofs regions. Nucleotide sequences of chromosomal regions, which included the ofs gene fragments of strain 89/1591, were retrieved from the National Center for Biotechnology Information website (http://www.ncbi.nlm.nih.gov) using the BLASTP program with the OFS amino acid sequence of $S$. suis strain 10 (GenBank/EMBL/DDBJ accession no. AAX56334).

For sequence determination of the ofs regions of NIAH 11433, MNCM51, DAT246, DAT252 and DAT299, PCR products amplified by primers Ofs-F and Ofs-R2 were sequenced by primer walking, and BLAST searches (http://www.ncbi.nlm.nih.gov/BLAST) were performed using the determined sequences. Because the sequence of the PCR product of NIAH 11433 was identical to that of the corresponding ofs region of $S$. suis strain 10, primers Ofs-AF (5'-ACTTCTTCGATTACACGTCC- $\left.3^{\prime}\right)$ and Ofs-AR (5'-CCATCACTATTATAGGCATAATCTC- $3^{\prime}$ ) were constructed on the basis of the sequence of strain 10 ofs. The whole ofs gene of NIAH 11433 was amplified, and the resultant $2.95 \mathrm{~kb}$ PCR product was sequenced by primer walking. For further sequencing of the upstream region of the ofs locus of NIAH 11433, an additional primer, Ofs-sq7 (5'-GAAAATACCTAAAGAAGGGCG-3'), was designed from the upstream sequence of the strain 10 ofs. The chromosomal region containing the upstream region of the ofs locus of NIAH 11433 was amplified with primers Ofs-sq7 and Ofs-R2, and the resultant PCR product was sequenced with primer Ofs-sq7.

Because BLAST searches showed that the sequence of the PCR product of MNCM51 was almost identical to those of the corresponding of $s$ regions of strain 89/1591, the whole ofs gene of MNCM51 was amplified with primers Ofs-AF2 (5' -TCCCAAAAGCTCTACGCTTC- (a)

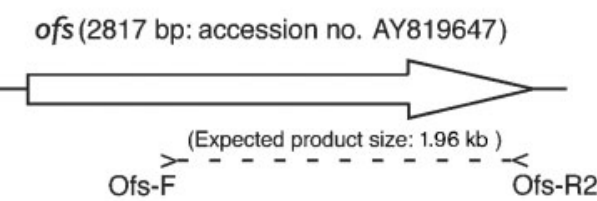

(b)

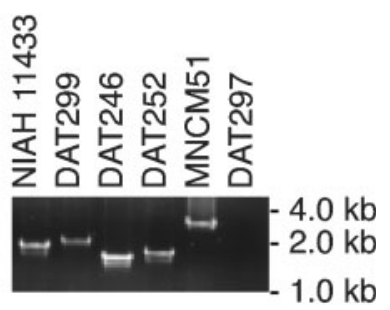

Fig. 1. (a) The positions of primers used for PCR amplification of ofs internal fragments. (b) PCR amplification of ofs fragments with primers Ofs- $F$ and Ofs-R2: the results from six representative isolates producing fragments of distinct sizes are shown, molecular sizes $(\mathrm{kb})$ are indicated on the right. 
$\left.3^{\prime}\right)$ and Ofs-AR2 (5' -TTCAGCCTCTGCAGCTATTG-3'), which were designed from the ofs sequences of strain 89/1591. The resultant $5.0 \mathrm{~kb}$ PCR product was then sequenced by primer walking.

For sequencing the ofs regions of DAT246, DAT252 and DAT299, primers Ofs-IV1 (5' -ATGTAGACTCCCCTTGTGAC-3') and Ofs-IV2 (5'-GATTCAGATAACTCTGCTCC-3') were designed for inverse PCR on the basis of the sequences of the PCR products amplified by primers Ofs-F and Ofs-R2. DNA fragments of about 11.0, 9.0 and $9.0 \mathrm{~kb}$ were amplified with primers Ofs-IV1 and Ofs-IV2 from PstIdigested and self-ligated genomic DNA of DAT246 and DAT252, and StuI-digested and self-ligated genomic DNA of DAT299, respectively. The products were then sequenced by primer walking. Signal sequences of the OFS proteins deduced from the determined sequences were predicted by the SignalP program (http://www.cbs. dtu.dk/services/SignalP).

Detection of serum opacification activity. The $1 \%$ SDS extraction technique, which has been described elsewhere (Baums et al., 2006), was used with the following modifications. A $1 \mathrm{ml}$ aliquot of midexponential phase culture $\left(\mathrm{OD}_{600}=0.3-0.4\right)$ was inoculated into $40 \mathrm{ml}$ fresh THY broth. After $4 \mathrm{~h}$ of growth at $37{ }^{\circ} \mathrm{C}, 160 \mu \mathrm{l}$ protease inhibitor cocktail (Sigma) was added to the cultures. Incubation at $37{ }^{\circ} \mathrm{C}$ was continued for an additional $2 \mathrm{~h}$, and the bacterial cells were collected by centrifugation. The pellet was resuspended in $300 \mu \mathrm{l}$ prewarmed $\left(42{ }^{\circ} \mathrm{C}\right) 1 \%$ SDS and rotated end-over-end at $37^{\circ} \mathrm{C}$ for $1 \mathrm{~h}$. For the detection of serum opacification activity of secreted proteins, the culture supernatants were filtered with a Millex-GV sterilizing filter unit with a $0.22 \mu \mathrm{m}$ pore size (Millipore) and placed on ice until use. A $25 \mu \mathrm{l}$ aliquot of the SDS extract or the filtered culture supernatant was added to $100 \mu$ horse serum (Sigma) in the wells of 96-well plates and mixed. As negative controls, $1 \%$ SDS or THY broth was added to the respective wells. The sealed plates were incubated with moderate shaking at $37{ }^{\circ} \mathrm{C}$ for $40 \mathrm{~h}$. After the addition of $100 \mu \mathrm{l} 0.9 \% \mathrm{NaCl}$, the $A_{450}$ value was measured. Differences in serum opacification activity were compared by unpaired $t$-test using Welch's modification.

Multilocus sequence typing (MLST). MLST was performed by sequencing seven housekeeping genes as described by King et al. (2002). The allelic numbers and sequence types (STs) of the isolates were determined by comparing their sequences with those in the $S$. suis MLST database (http://ssuis.mlst.net). Novel alleles and STs were assigned through submission of the respective data to the database. Analysis of ST complexes was performed with the eBURST program (http://eburst.mlst.net) (Feil et al., 2004).

\section{RESULTS AND DISCUSSION}

\section{Variation of the ofs gene}

Oligonucleotide primers Ofs-F and Ofs-R2 were expected to amplify a $1.96 \mathrm{~kb}$ fragment from the ofs gene of $S$. suis strain 10 (Fig. 1a). From genomic DNA samples of 29 of the 108 isolates, a PCR product of the expected size was amplified, while 3, 41, 9 and 15 isolates generated products with sizes of approximately $2.1 \mathrm{~kb}, 1.6 \mathrm{~kb}, 1.8 \mathrm{~kb}$ and $3.3 \mathrm{~kb}$, respectively. From the remaining 11 isolates, including strain $89 / 1591$, no specific PCR products were amplified (Fig. 1b). Since the results showed sequence divergence of the ofs genes among the S. suis isolates, we selected five representative isolates, NIAH 11433, DAT299, DAT246, DAT252 and MNCM51, which generated 1.96, 2.1, 1.6, 1.8 and $3.3 \mathrm{~kb}$ products, respectively, and determined the nucleotide sequences of their chromosomal regions corresponding to the ofs locus. In addition, BLAST searches were performed using genome sequencing data of strain 89/1591, and the sequences of two distinct chromosomal regions, which were highly homologous to the $5^{\prime}$ and $3^{\prime}$ regions of the ofs sequence of strain 10 , were retrieved.

As shown in Fig. 2, the sequence analysis identified several allelic variants of ofs. The ofs gene of NIAH 11433 (designated type-1 ofs) was $2817 \mathrm{bp}$ in length, and the nucleotide sequence was completely identical to that of strain 10 (Baums et al., 2006). The ofs variant of DAT299 (designated type-2 ofs) was $3177 \mathrm{bp}$ in length and encoded a protein of 1058 amino acids. Similar to type-1 OFS, type2 OFS also consisted of a signal sequence, a large $\mathrm{N}$ terminal domain, repetitive sequence elements and a Cterminal cell wall sorting signal (Fig. 2). The deduced amino acid sequence of type-2 OFS was $79.0 \%$ identical and $84.9 \%$ similar to that of type-1 OFS. However, due to mutations, the position of the start codon of type- 2 ofs was different from that of type-1 ofs, resulting in alteration of the signal sequence (Fig. 3). In addition, type-1 OFS possessed three repetitive sequence elements, while type-2 OFS possessed four repetitive sequence elements (Fig. 2).

The ofs regions of DAT246 and DAT252, which generated PCR products of 1.6 and $1.8 \mathrm{~kb}$, respectively, showed similar genetic organizations (Fig. 2). In both regions, ORFs were shifted due to a deletion of an adenine residue between nucleotide positions 625 and 630 , resulting in the occurrence of a stop codon at nucleotide positions 649651. The ofs variants of the two strains encoded the same proteins of 216 amino acids, and therefore both of the genes were designated type-3 ofs. The deduced amino acid sequence of type-3 OFS was 54.2 and $85.6 \%$ identical to those of type-1 and type-2 OFS, respectively.

In MNCM51, the ofs gene was disrupted by the insertion of an IS element. The gene (designated type 4) was supposed to produce a protein of 564 amino acids (Fig. 2), and the deduced amino acid sequence was 70.3 and $84.6 \%$ identical to those of type-1 and type-2 OFS, respectively. Strain 89/1591 also has a similar gene whose nucleotide sequence was completely identical to that of type- 4 ofs of MNCM51. However, in 89/1591, the genetic region was separated by an IS element and was translocated (Fig. 2).

These results demonstrated that in S. suis there are at least three allelic variants of ofs in addition to the originally reported ofs gene. However, because type- 3 and type- 4 ofs were interrupted by mutations, only type- 1 and type- 2 ofs seem to produce intact OFS proteins. We hereafter refer to the isolates that produced PCR fragments of 1.96, 2.1 and $3.3 \mathrm{~kb}$ with primers Ofs-F and Ofs-R2 as isolates with type1 , type-2 and type- 4 ofs, respectively. The isolates that generated PCR products of either 1.6 or $1.8 \mathrm{~kb}$ are referred to as isolates with type- 3 ofs. In addition, the isolates that did not produce any specific PCR products were further analysed by Southern blot hybridization analysis. Using 
Strain PCR product size with

NIAH 11433

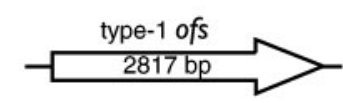

$\mathrm{N}$-terminal domain (25-673) R1-R3 $\Delta(674-830)$

$1960 \mathrm{bp}$

$817 \mathrm{~b}$

(938 aa)

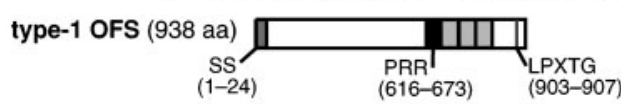

DAT299

$2113 \mathrm{bp}$

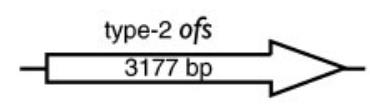

$\mathrm{N}$-terminal domain (39-739) R1-R4 $\Delta$ (740-950)

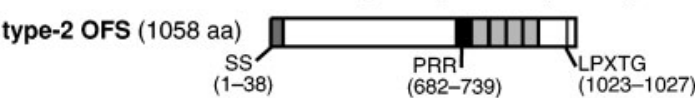

DAT246

1627 bp

DAT252

$1786 \mathrm{bp}$
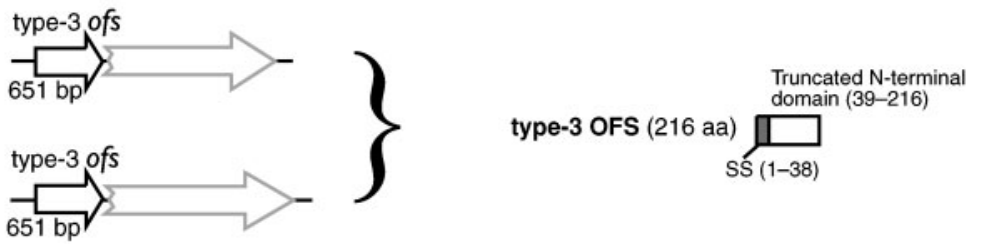

MNCM51

$3288 \mathrm{bp}$

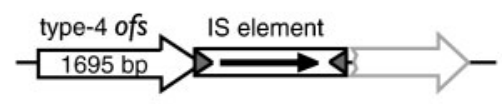

$89 / 1591$

Not amplified
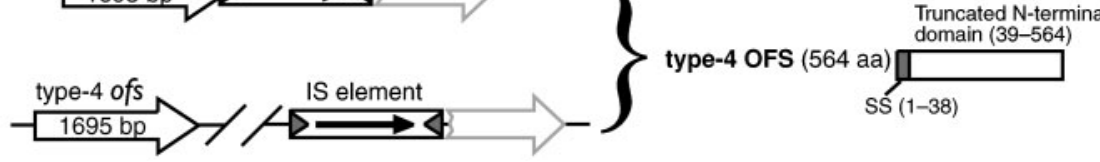

Fig. 2. Genetic map of the ofs regions of six representative isolates and diagrams of the putative OFS variants. Arrows drawn with grey lines indicate pseudogenes corresponding to residual 3' parts of ofs, which are not supposed to be translated. PRR, Proline-rich region; $\mathrm{Rn}$, repetitive sequence element; SS, putative $\mathrm{N}$-terminal signal sequence.

ClaI-digested genomic DNA and the ofs probe prepared from type-1 ofs, they were classified as of $s$-negative isolates and isolates with type- 4 ofs (see Supplementary Fig. S1 and Supplementary Table S1 available with the online journal).

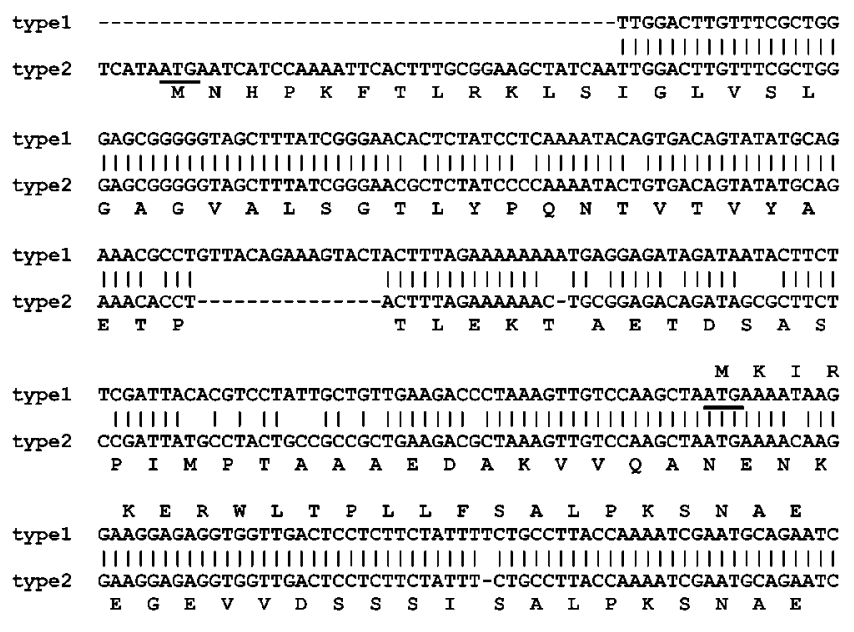

Fig. 3. Comparison of nucleotide sequences between the $5^{\prime}$ regions of type- 1 ofs of NIAH 11433 and type-2 ofs of DAT299, and their deduced amino acid sequences.

\section{Serum opacification activity of OFS and its variants}

SDS extracts and culture supernatants of eight representative isolates were tested for their ability to opacify a serum. As speculated from the sequence data, only the isolates with type- 1 ofs and type-2 ofs showed surfaceassociated serum opacification activity (Fig. 4a). The opacification activities of the isolates were significantly higher $(P<0.05)$ than those of the isolates with type-3 ofs or type- 4 ofs, indicating that only type- 1 and type- 2 ofs can express functional OFS on the cell surface.

Baums et al. (2006) reported that concentrated culture supernatants of S. suis strain 10, which possesses type-1 ofs, were negative for serum opacification. In agreement with this, neither P1/7 nor NIAH 11433 showed serum opacification activity in the culture supernatant (Fig. 4b). Interestingly, DAT289 and DAT299, which possess type-2 $o f s$, showed strong serum opacification activity in their culture supernatants (Fig. 4b). Although we do not have any evidence to predict the mechanism, the type- 2 OFS specific structures described above may affect the stability of the proteins in the culture supernatant and/or the secretion of the proteins into the supernatant.

In $S$. pyogenes, expression of sof is positively regulated by the multiple-gene regulator Mga (formerly designated VirR), which also co-regulates the expression of emm, 
(a)

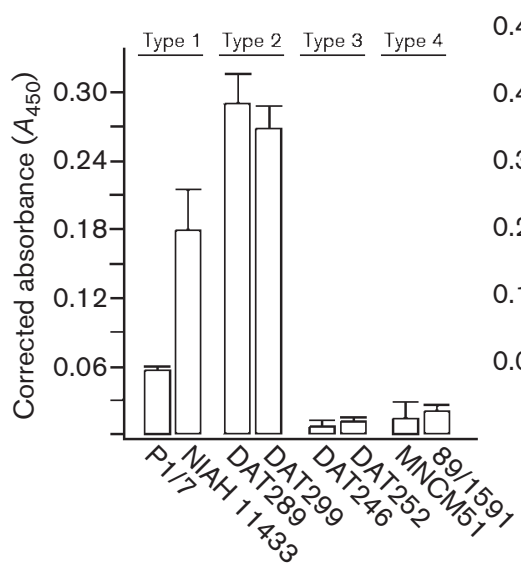

(b)

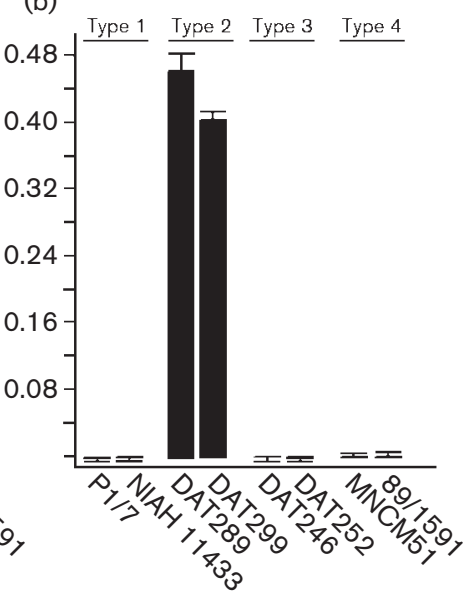

Fig. 4. Serum opacification activity of $S$. suis isolates with distinct ofs genotypes. The $1 \%$ SDS extracts (a) or culture supernatants (b) of the isolates were tested for serum opacification activity with a microwell plate assay as described in Methods. The absorbance of serum incubated with $1 \%$ SDS alone (a) or THY broth alone (b) was subtracted from each value (corrected absorbance). Mean values and SDS of three or four independent experiments, each performed in triplicate, are shown. encoding $M$ protein, and $\operatorname{scp} A$, encoding the C5a peptidase (Podbielski et al., 1992; McLandsborough \& Cleary, 1995). However, it is unknown how S. suis regulates the expression of ofs. Intriguingly, even though the deduced amino acid sequences of type-1 OFS in P1/7 and NIAH 11433 are identical to each other (D. Takamatsu, M. Osaki \& T. Sekizaki, data not shown), the serum opacification activity of the SDS extract of P1/7 was significantly lower than that of NIAH $11433(P=0.0280)$ (Fig. 4a). Although the difference in the serum opacification activities could be caused by differences in the extraction efficiency of surfaceassociated proteins between the two strains, S. suis may have some regulatory mechanisms for the expression of ofs.

\section{Correlation of the ofs genotypes with MLST results}

As shown in Table 1, regardless of their origin, approximately $30 \%$ of the isolates tested possessed either type- 1 or type-2 ofs. However, isolates with type- 2 ofs were recovered only from three healthy pigs. By means of MLST analysis using representative isolates, a strong association between the ST1 complex and isolates with type- 1 ofs was observed. In addition, the majority of isolates with type-3 ofs and type- 4 ofs were assigned to the ST27 complex. On the other hand, isolates with type- 2 ofs were not related to known ST complexes (Table 2).
In S. suis, more than 400 isolates have been analysed by MLST, and 3 major clonal complexes have been identified so far (the ST1, ST27 and ST87 complexes) (King et al., 2002; Chang et al., 2006; Ye et al., 2006; Rehm et al., 2007). Among the three complexes, the ST1 complex was strongly associated with isolates from pigs with classical invasive diseases, including septicaemia and meningitis (King et al., 2002). In addition, the majority of human clinical isolates, including those of the outbreak in Sichuan, China, in 2005, belonged to the ST1 complex (King et al., 2002; Chang et al., 2006; Ye et al., 2006). The ST27 and ST87 complexes contained significantly higher numbers of swine lung isolates (King et al., 2002).

In our previous report, we superimposed the distribution of the virulence-associated genes $\operatorname{mrp}$ (encoding MRP), epf (encoding EF) and sly (encoding suilysin) on the MLST data, and found that all the isolates assigned to the ST1 complex were positive for $\operatorname{mrp}$ (or its variant), epf (or its variant) and sly. However, the isolates assigned to the ST27 complex did not possess one or more of these genes (Takamatsu et al., 2008). Similar data were also reported by Rehm et al. (2007). Among the isolates they analysed, all the isolates assigned to ST1 were positive for mrp, epf and sly, while the other isolates, except for one, lacked one or more of these genes. These results suggest that mrp, epf and sly can be used as epidemiological markers for ST1 complex members. However, because mrp, epf or sly mutants have been shown

Table 1. Prevalence of type-1 and type-2 ofs genes among the isolates used in this study

Bold text indicates results of particular interest.

\begin{tabular}{|lrlll|}
\hline Origin of isolates & \multicolumn{3}{c|}{ No. of isolates $\mathbf{( \% )}$} & Total \\
\cline { 2 - 4 } & \multicolumn{1}{c}{ Type $\mathbf{1}$} & Type 2 & Other \\
\hline Diseased pigs & $\mathbf{1 7}(\mathbf{2 8 . 3} \%)$ & $0(0 \%)$ & $43(71.7 \%)$ & $60(100 \%)$ \\
Healthy pigs & $\mathbf{3 ( 1 4 . 3 \% )}$ & $\mathbf{3}(\mathbf{1 4 . 3} \%)$ & $15(71.4 \%)$ & $21(100 \%)$ \\
Human patients & $\mathbf{9 ( 3 3 . 3 \% )}$ & $0(0 \%)$ & $18(66.7 \%)$ & $27(100 \%)$ \\
\hline
\end{tabular}


Table 2. Association of MLST typing results with ofs genotypes

\begin{tabular}{|c|c|c|c|}
\hline ofs genotype & Isolate/strain & Origin & ST $(\text { ST complex })^{*}$ \\
\hline \multirow[t]{10}{*}{ Type 1} & $\mathrm{P} 1 / 7$ & Diseased pig & $1(1)$ \\
\hline & NIAH 11433 & Diseased pig & $1(1)$ \\
\hline & D282 & Diseased pig & $1(1)$ \\
\hline & DAT294 & Healthy pig & 112 (unrelated) \\
\hline & DAT300 & Healthy pig & 115 (unrelated) \\
\hline & $\mathrm{H} 11 / 1$ & Human & $1(1)$ \\
\hline & MNCM01 & Human & $1(1)$ \\
\hline & MNCM06 & Human & $1(1)$ \\
\hline & MNCM07 & Human & $11(1)$ \\
\hline & MNCM16 & Human & $1(1)$ \\
\hline \multirow[t]{3}{*}{ Type 2} & DAT289 & Healthy pig & 110 (unrelated) \\
\hline & DAT291 & Healthy pig & 111 (unrelated) \\
\hline & DAT299 & Healthy pig & 114 (unrelated) \\
\hline \multirow[t]{5}{*}{ Type 3} & DAT246 & Diseased pig & $28(27)$ \\
\hline & DAT252 & Diseased pig & $108(64)$ \\
\hline & MNCM21 & Human & $101(27 \dagger)$ \\
\hline & MNCM43 & Human & $28(27)$ \\
\hline & MNCM50 & Human & $104(27 \dagger)$ \\
\hline \multirow[t]{15}{*}{ Type 4} & TD10 & Healthy pig & $25(27 \dagger)$ \\
\hline & MNCM04 & Human & $25(27 \dagger)$ \\
\hline & MNCM10 & Human & $25(27 \dagger)$ \\
\hline & MNCM24 & Human & $25(27 \dagger)$ \\
\hline & MNCM25 & Human & $102(27 \dagger)$ \\
\hline & MNCM26 & Human & $25(27 \dagger)$ \\
\hline & MNCM33 & Human & $103(27 \dagger)$ \\
\hline & MNCM51 & Human & $25(27 \dagger)$ \\
\hline & MNCM54 & Human & $102(27 \dagger)$ \\
\hline & MNCM55 & Human & $25(27 \dagger)$ \\
\hline & LPH3 & Human & $103(27 \dagger)$ \\
\hline & LPH4 & Human & $25(27 \dagger)$ \\
\hline & LPH5 & Human & $103(27 \dagger)$ \\
\hline & LPH12 & Human & $25(27 \dagger)$ \\
\hline & $98-3634$ & Human & $25(27 \dagger)$ \\
\hline \multirow[t]{3}{*}{ ofs negative } & DAT288 & Healthy pig & 109 (unrelated) \\
\hline & DAT297 & Healthy pig & 113 (unrelated) \\
\hline & DAT301 & Healthy pig & 116 (unrelated) \\
\hline
\end{tabular}

*The STs of NIAH 11433, DAT246, DAT252, DAT288, DAT289, DAT291, DAT294, DAT297, DAT299, DAT300 and DAT301 were determined in this study. MLST data for the other isolates were retrieved from the S. suis MLST database (http://ssuis.mlst.net).

$\nmid$ ST25, ST101, ST102, ST103 and ST104 belong to the ST27 complex, only with a less-stringent approach that defines an ST complex by the sharing of alleles at five or more of the seven loci.

to be as virulent as the respective wild-type strains after experimental infection of newborn or young pigs (Smith et al., 1996; Lun et al., 2003), it is unclear whether or not these markers have some role in the pathogenesis of the ST1 complex members. On the contrary, severe attenuation in virulence of an ofs mutant was demonstrated by an experimental infection model in piglets, and ofs was suggested to be an important virulence determinant (Baums et al., 2006). These results, together with the present findings, suggest that type-1 OFS is one of the important virulence factors that may confer upon the ST1 complex members an ability to cause invasive diseases.
SOF of S. pyogenes has been established as a virulence determinant (Courtney et al., 1999). Disruption of highdensity lipoprotein (HDL), which is an anti-inflammatory and anti-atherogenic factor, by SOF is suggested to contribute to the pathogenesis of $S$. pyogenes infection (Courtney et al., 2006). Although it is unknown how OFS is involved in the pathogenesis of $S$. suis, a similar mechanism may contribute to the development of invasive diseases by this organism. However, in our data, approximately $70 \%$ of the S. suis isolates from diseased pigs and human patients did not possess functional ofs genes (Table 1). In addition, genes encoding type-2 OFS showing 
higher opacification activities than type-1 OFS were found only in isolates from healthy pigs. These results indicate that OFS is not an essential nor a sufficient factor for S. suis isolates to cause invasive diseases. For further comprehensive understanding of the pathogenesis of S. suis, identification of additional virulence determinants and comparison of their distribution with the MLST dataset will be needed.

\section{ACKNOWLEDGEMENTS}

This study was supported by a Grant-in-Aid from the Zoonoses Control Project of the Ministry of Agriculture, Forestry and Fisheries of Japan, and the Endowment Fund for Medical Research, Faculty of Medicine, Chiang Mai University. We thank Marcelo Gottschalk for kindly supplying North American and European S. suis strains. This publication made use of the $S$. suis multilocus sequence typing website (http://ssuis.mlst.net) curated by Adrian Whatmore. This site is hosted at Imperial College and development is funded by the Wellcome Trust.

\section{REFERENCES}

Arends, J. P. \& Zanen, H. C. (1988). Meningitis caused by Streptococcus suis in humans. Rev Infect Dis 10, 131-137.

Baums, C. G., Kaim, U., Fulde, M., Ramachandran, G., Goethe, R. \& Valentin-Weigand, P. (2006). Identification of a novel virulence determinant with serum opacification activity in Streptococcus suis. Infect Immun 74, 6154-6162.

Chang, B., Wada, A., Ikebe, T., Ohnishi, M., Mita, K., Endo, M., Matsuo, H., Asatuma, Y., Kuramoto, S. \& other authors (2006). Characteristics of Streptococcus suis isolated from patients in Japan. Jpn J Infect Dis 59, 397-399.

Courtney, H. S., Hasty, D. L., Li, Y., Chiang, H. C., Thacker, J. L. \& Dale, J. B. (1999). Serum opacity factor is a major fibronectin-binding protein and a virulence determinant of $\mathrm{M}$ type 2 Streptococcus pyogenes. Mol Microbiol 32, 89-98.

Courtney, H. S., Zhang, Y.-M., Frank, M. W. \& Rock, C. O. (2006). Serum opacity factor, a streptococcal virulence factor that binds to apolipoproteins A-I and A-II and disrupts high density lipoprotein structure. J Biol Chem 281, 5515-5521.

Feil, E. J., Li, B. C., Aanensen, D. M., Hanage, W. P. \& Spratt, B. G. (2004). евURST: inferring patterns of evolutionary descent among clusters of related bacterial genotypes from multilocus sequence typing data. J Bacteriol 186, 1518-1530.

Gottschalk, M., Lebrun, A., Wisselink, H., Dubreuil, J. D., Smith, H. \& Vecht, U. (1998). Production of virulence-related proteins by Canadian strains of Streptococcus suis capsular type 2. Can J Vet Res 62, 75-79.

Jacobs, A. A. C., Loeffen, P. L. W., van den Berg, A. J. G. \& Storm, P. K. (1994). Identification, purification, and characterization of a thiolactivated hemolysin (suilysin) of Streptococcus suis. Infect Immun 62, $1742-1748$.
King, S. J., Leigh, J. A., Heath, P. J., Luque, I., Tarradas, C., Dowson, C. G. \& Whatmore, A. M. (2002). Development of a multilocus sequence typing scheme for the pig pathogen Streptococcus suis: identification of virulent clones and potential capsular serotype exchange. J Clin Microbiol 40, 3671-3680.

Lun, S., Perez-Casal, J., Connor, W. \& Willson, P. J. (2003). Role of suilysin in pathogenesis of Streptococcus suis capsular serotype 2. Microb Pathog 34, 27-37.

McLandsborough, L. A. \& Cleary, P. P. (1995). Insertional inactivation of virR in Streptococcus pyogenes M49 demonstrates that VirR functions as a positive regulator of ScpA, FcRA, OF, and M protein. FEMS Microbiol Lett 128, 45-51.

Osaki, M., Takamatsu, D., Tsuji, N. \& Sekizaki, T. (2000). Cloning and characterization of the gene encoding $O$-acetylserine lyase from Streptococcus suis. Curr Microbiol 40, 67-71.

Podbielski, A., Kaufhold, A. \& Lütticken, R. (1992). The vir-regulon of Streptococcus pyogenes: coordinate expression of important virulence factors. Immun Infekt 20, 161-168.

Rehm, T., Baums, C. G., Strommenger, B., Beyerbach, M., ValentinWeigand, P. \& Goethe, R. (2007). Amplified fragment length polymorphism of Streptococcus suis strains correlates with their profile of virulence-associated genes and clinical background. J Med Microbiol 56, 102-109.

Sekizaki, T., Otani, Y., Osaki, M., Takamatsu, D. \& Shimoji, Y. (2001). Evidence for horizontal transfer of $S s u$ DAT1I restriction-modification genes to the Streptococcus suis genome. J Bacteriol 183, 500-511.

Smith, H. E., Vecht, U., Wisselink, H. J., Stockhofe-Zurwieden, N., Biermann, Y. \& Smits, M. A. (1996). Mutants of Streptococcus suis types 1 and 2 impaired in expression of muramidase-released protein and extracellular protein induce disease in newborn germfree pigs. Infect Immun 64, 4409-4412.

Smith, H. E., Damman, M., van der Velde, J., Wagenaar, F., Wisselink, H. J., Stockhofe-Zurwieden, N. \& Smits, M. A. (1999). Identification and characterization of the cps locus of Streptococcus suis serotype 2: the capsule protects against phagocytosis and is an important virulence factor. Infect Immun 67, 1750-1756.

Staats, J. J., Feder, I., Okwumabua, O. \& Chengappa, M. M. (1997). Streptococcus suis: past and present. Vet Res Commun 21, 381-407.

Takamatsu, D., Wongsawan, K., Osaki, M., Nishino, H., Ishiji, T., Tharavichitkul, P., Khantawa, B., Fongcom, A., Takai, S. \& Sekizaki, T. (2008). Streptococcus suis in humans, Thailand. Emerg Infect Dis 14, 181-183.

Vecht, U., Arends, J. P., van der Molen, E. J. \& van Leengoed, L. A. (1989). Differences in virulence between two strains of Streptococcus suis type II after experimentally induced infection of newborn germfree pigs. Am J Vet Res 50, 1037-1043.

Vecht, U., Wisselink, H. J., Jellema, M. L. \& Smith, H. E. (1991). Identification of two proteins associated with virulence of Streptococcus suis type 2. Infect Immun 59, 3156-3162.

Vecht, U., Wisselink, H. J., van Dijk, J. E. \& Smith, H. E. (1992). Virulence of Streptococcus suis type 2 strains in newborn germfree pigs depends on phenotype. Infect Immun 60, 550-556.

Ye, C., Zhu, X., Jing, H., Du, H., Segura, M., Zheng, H., Kan, B., Wang, L., Bai, X. \& other authors (2006). Streptococcus suis sequence type 7 outbreak, Sichuan, China. Emerg Infect Dis 12, 1203-1208. 